\title{
Modification of the order of the magnetic phase transition in cobaltites without changing their crystal space group
}

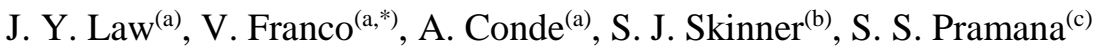 \\ (a) Dpto. Física de la Materia Condensada, ICMSE-CSIC, Universidad de Sevilla, Apdo. \\ 1065. 41080-Sevilla, Spain \\ (b) Department of Materials, Imperial College London, Exhibition Road, London, SW7 \\ 2AZ, United Kingdom \\ (c) School of Engineering, Newcastle University, Newcastle upon Tyne, NE1 7RU, \\ United Kingdom \\ * corresponding author email: vfranco@us.es
}

It has been found that $\mathrm{GdBa}_{1-\mathrm{x}} \mathrm{Sr}_{\mathrm{x}} \mathrm{Co}_{2} \mathrm{O}_{6-\delta}$ can exhibit consecutive magnetic transitions: antiferromagnetic-ferromagnetic (AFM-FM) transition followed by ferromagnetic-paramagnetic transition (FM-PM), which give rise to a coexistence of inverse and conventional magnetocaloric effect (MCE), respectively. In the pristine compound ( $\mathrm{x}=0$ ), its AFM-FM transition is shown to belong to a first-order phase transition and the FM-PM to a second-order type. Despite it is widely known that the properties of cobaltites are highly influenced by their oxygen content and type of doping carriers, in this work, further evaluation using magnetocaloric analysis (universal curve method and a quantitative criterion using magnetic field dependence of the magnetic entropy change) reveals that the first-order AFM-FM phase transition converts into a second-order character with just $\mathrm{Sr}$ doping of $\mathrm{x}=0.1$ (despite of having the same space group at room temperature and type of dopant carrier as $\mathrm{x}=0$ ), severely affecting its thermomagnetic properties. Moreover, the peaks of these two MCE span over a temperature range that is larger than those reported for cobaltite-type materials, making it closer to room temperature applications. 


\section{Introduction}

$\mathrm{Ln}_{1-\mathrm{x}} \mathrm{A}_{\mathrm{x}} \mathrm{CoO}_{3-\delta}$ perovskite cobaltites (Ln: lanthanides, A: alkaline-earth metals) are known for their applications in magnetodielectric materials, solid oxide fuel cells, electrocatalytic activity as well as giant magnetoresistance effects etc. [1-6]. Their performance is related to details of their phase transitions, which can also be highly compositional dependent [7]. The oxygen content (3- $\delta$ ) is mainly dictated by the dopant level $\mathrm{x}\left(\mathrm{Ln}^{3+}+1 / 2 \mathrm{O}^{2-} \rightarrow \mathrm{A}^{2+}+1 / 2 \square\right)$ and partitioning of $\mathrm{Co}^{2+} / \mathrm{Co}^{3+} / \mathrm{Co}^{4+}$, leading to the possibility of electron or hole doping into the systems [8]. Among $\operatorname{Ln}_{1-\mathrm{x}} \mathrm{A}_{\mathrm{x}} \mathrm{CoO}_{3}$ cobaltites, the ordered oxygen vacancy double perovskite, $\mathrm{GdBaCo}_{2} \mathrm{O}_{6-\delta}$ (Fig. 1) exhibits thermomagnetic transitions and properties that are dependent on their $\delta$ values: for $\delta>0.5$, it corresponded to electron doping while $\delta<0.5$ to hole doping [9]. Recently, it has been reported that $\mathrm{Sr}$ doping in $\mathrm{GdBa}_{1-\mathrm{x}} \mathrm{Sr}_{\mathrm{x}} \mathrm{Co}_{2} \mathrm{O}_{6-\delta}$ led to an improvement in conductivity due to the effects of hole doping from lowered oxygen deficiency with increasing Sr content [10].

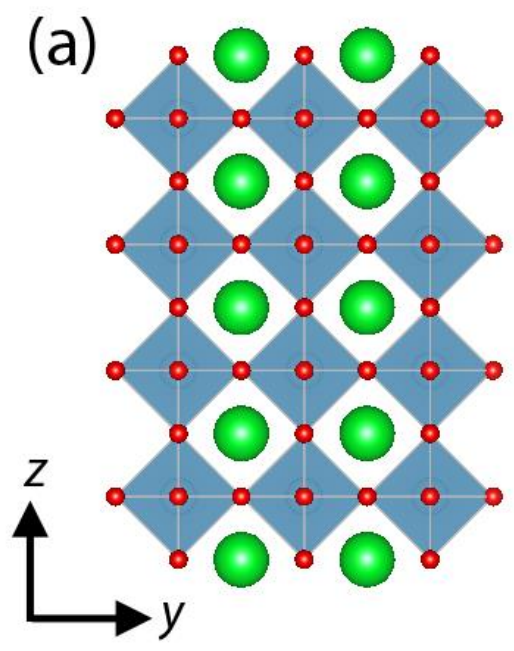

(b)

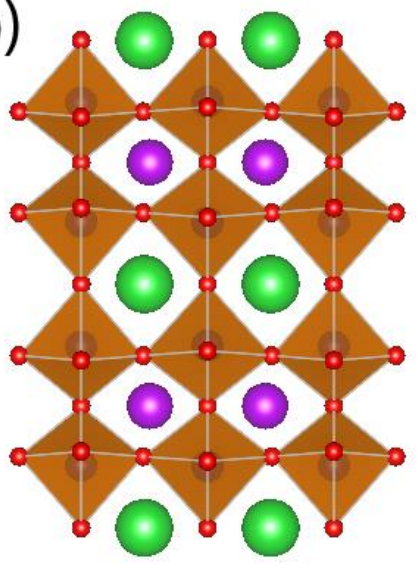

(c)

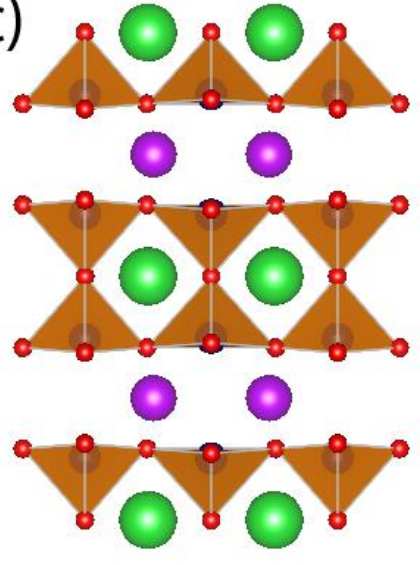

Fig. 1: Crystal structure representation of (a) a single perovskite $\mathrm{ABO}_{3}$, where $\mathrm{A}$ (green) is located in the centre of corner-connected $\mathrm{BO}_{6}$ octahedra (blue); (b) an ordered perovskite $\mathrm{AA}^{\prime} \mathrm{B}_{2} \mathrm{O}_{6}$, where $\mathrm{A}$ (green) and $\mathrm{A}^{\prime}$ (purple) stack in an alternate sequence along z-crystallographic axis with cornerconnected $\mathrm{BO} 6$ octahedra shown in brown, for example $\mathrm{GdBaCo}_{2} \mathrm{O}_{6}$; (c) defective ordered perovskite $\mathrm{AA}^{\prime} \mathrm{B}_{2} \mathrm{O}_{6-\delta}$, where the vacancy of oxygen $\left(\delta\right.$, red) is preferentially created in the $x y$-plane in the $\mathrm{A}^{\prime}$ layer. 
Similar compounds, like manganites, have been reported as potential magnetocaloric material candidates. Their doping dependencies on their phase transitions, transition temperatures as well as the temperature range of magnetic refrigeration have been widely reported [11], similar to perovskite cobaltites. There are only very few magnetocaloric effect (MCE) studies in cobaltites, which are usually focused on $\mathrm{La}_{1-\mathrm{x}} \mathrm{Sr}_{x} \mathrm{CoO}_{3}, \mathrm{Pr}_{1-\mathrm{x}} \mathrm{Sr}_{x} \mathrm{CoO}_{3}$ and $\mathrm{Nd}_{1-\mathrm{x}} \mathrm{Sr}_{x} \mathrm{CoO}_{3}$ systems[12-14]. However, reports on cobaltites with $\mathrm{Ln}=\mathrm{Gd}$ are scarce, whereby only one study reports theoretical simulations of $\mathrm{Gd}_{1-\mathrm{x}} \mathrm{Ca}_{x} \mathrm{BaCo}_{2} \mathrm{O}_{5.5}$ [15] while experimental results were shown only for $\mathrm{Gd}_{0.7} \mathrm{Sr}_{0.3} \mathrm{CoO}_{3}$ [16] (both with $\mathrm{Ln}=\mathrm{Gd}$ ), and none in particular about $\mathrm{GdBa}_{1-\mathrm{x}} \mathrm{Sr}_{\mathrm{x}} \mathrm{Co}_{2} \mathrm{O}_{6-\delta}$. In addition, the latter's thermomagnetic behavior and the magnetic field dependence of magnetocaloric response have not been reported. In this work, we aim to use magnetocaloric studies to further evaluate these materials, which reveal that $\mathrm{Sr}$ substitutions can drastically alter the magnetic behavior and its phase transitions. In particular, we have found that the pristine sample exhibits an inverse MCE then closely accompanied by a conventional MCE, whereby the former arises from the first-order antiferromagnetic-ferromagnetic (AFM-FM) phase transition and the latter from a ferromagnetic-paramagnetic (FM-PM) transition. The inverse MCE of this material has not been reported as the only related work, theoretical simulations on $\mathrm{Gd}_{1-\mathrm{x}} \mathrm{Ca}_{\mathrm{x}} \mathrm{BaCo}_{2} \mathrm{O}_{5.5}[15]$, report solely on the conventional MCE. The relatively similar cobaltite (which is not a double perovskite), $\mathrm{Gd}_{0.7} \mathrm{Sr}_{0.3} \mathrm{CoO}_{3}$, showed a conventional MCE at $10 \mathrm{~K}$ (magnetic entropy change of $\Delta S_{M^{\sim}}-12 \mathrm{~J} \mathrm{~kg}^{-1} \mathrm{~K}^{-1}$ for $5 \mathrm{~T}$ ) [16], which is at much lower temperatures than our results. The two types of MCE observed occur at higher temperatures $(175-277 \mathrm{~K})$ that could be useful for paving the ground to discover new materials with potentially more alternative applications than room temperature magnetic refrigeration. Collectively, our findings show that magnetocaloric studies are useful to offer insight into the magnetic behavior of doped perovskites, as they reveal an alteration of the order of phase transition with just minor Sr doping of $\mathrm{x}=0.1$ despite the sample exhibiting same room temperature crystal symmetry and electron doping carrier as the pristine or undoped sample. Therefore, with apparently the same typical influences on the properties of cobaltites, their behavior can be severely affected. 


\section{Experimental}

The $\mathrm{GdBa}_{1-\mathrm{x}} \mathrm{Sr}_{\mathrm{x}} \mathrm{Co}_{2} \mathrm{O}_{6-\delta}$ powders were synthesized via a solid-state reaction whereby stoichiometric amounts of $\mathrm{Gd}_{2} \mathrm{O}_{3}$ (Alfa Aesar, 99.99\%), $\mathrm{BaCO}_{3}$ (Alfa Aesar, 99.8\%), $\mathrm{SrCO}_{3}$ (Alfa Aesar, 99.99\%) and $\mathrm{Co}_{3} \mathrm{O}_{4}$ (Alfa Aesar, 99.7\%) starting powders were mixed and ground without pre-calcination. They were sintered at $1100{ }^{\circ} \mathrm{C}$ for $60 \mathrm{~h}$ in static air with intermediate grinding for intervals of $12 \mathrm{~h}$. Their measured compositions, quantified using electron probe microanalysis (JEOL JXA-8530F equipped with five wavelength dispersive spectrometers), were within the standard deviation of the nominal values. In this work, the samples are denoted by their $\mathrm{Sr}$ content (i.e. $\mathrm{x}=0-0.5$ ) as: $\mathrm{Sr} 0, \mathrm{Sr} 1, \mathrm{Sr} 2, \mathrm{Sr} 3, \mathrm{Sr} 4$ and $\mathrm{Sr} 5$ respectively. The Rietveld analyses of powder X-ray diffraction results showed that high Sr content led to crystallization in higher symmetry in the crystal structures: (i) the starting orthorhombic symmetry with Pmmm space group with doubling in $b$ - and $c$-axes for $\operatorname{Sr} 0$ and $\operatorname{Sr} 1$ samples (Rietveld refinement of $\mathrm{Sr} 1$ is shown in Fig. 2 as an example, wherein $2 \theta_{001}=11.73^{\circ}$ indicates the doubling in $c$, and $2 \theta_{010}=11.33^{\circ}, 2 \theta_{130}=41.79^{\circ}$ and $2 \theta_{131}=43.56^{\circ}$ indicate the doubling in $b$ ) was transformed into (ii) Pmmm with only doubling in $c$-axis ( $\mathrm{Sr} 2$ and $\mathrm{Sr} 3$ ), then into (iii) tetragonal $P 4 / \mathrm{mmm}$ ( $\mathrm{Sr} 4$ and Sr5) [10]. The oxygen content calculated from the Co valence $\left(V_{C o}\right)$ from iodometric titration reveals that the oxygen deficiency decreases as $\mathrm{Sr}$ replaces $\mathrm{Ba}$, with $\delta=0.45(1)$ found for $\mathrm{Sr} 5$ (for all compositions, $V_{C o}$ values are summarized in Table 1). In addition, the calculations from the bond valence sum (BVS) analysis of cobalt-oxygen bonds (which indicates the bond strength in ionic compounds) were in agreement with the results from iodometric titration. Further details of the structural characterization and analysis, for both bulk and surface, are published elsewhere [10]. 


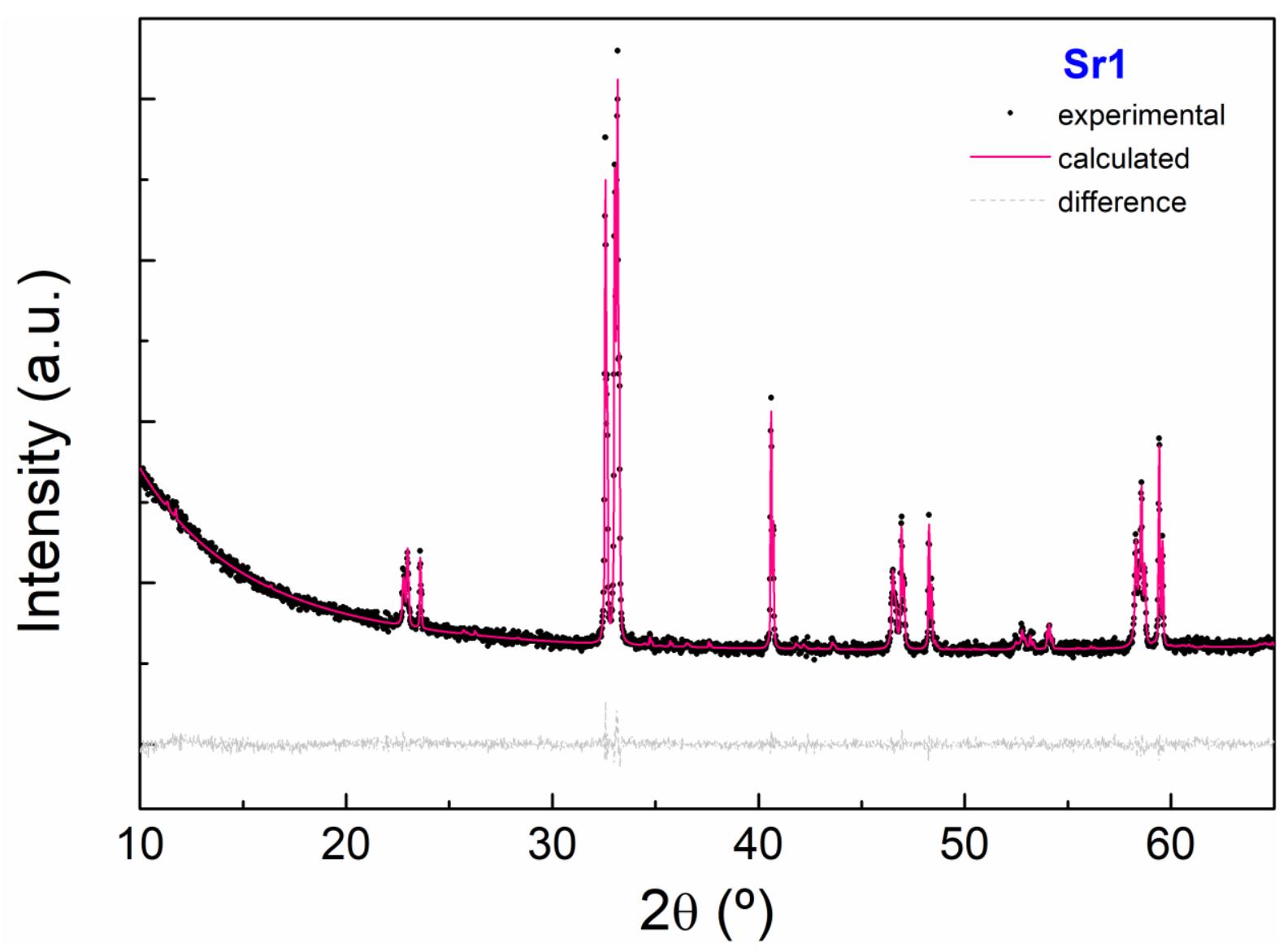

Fig. 2: Rietveld refinement of the XRD data of $\mathrm{Sr} 1$ sample performed at room temperature using $\mathrm{Cu}$ $K_{\alpha}$ radiation $\left(R_{w p}=0.029, R_{\text {Bragg }}=0.009\right)$. The doubling in its $c$-axis is indicated by $2 \theta_{001}=11.73^{\circ}$ and its doubling in $b$ by $2 \theta_{010}=11.33^{\circ}, 2 \theta_{130}=41.79^{\circ}$ and $2 \theta_{131}=43.56^{\circ}$.

Table 1. Oxygen content and cobalt valence states in $\mathrm{GdBa}_{1-\mathrm{x}} \mathrm{Sr}_{\mathrm{x}} \mathrm{Co}_{2} \mathrm{O}_{6-\delta}$

\begin{tabular}{|c|c|c|c|}
\hline Sr content $(\mathbf{x})$ & Sample & Oxygen content $(\mathbf{6}-\boldsymbol{\delta})$ & Cobalt Valence $\left(\mathbf{V}_{\mathbf{C}_{\mathbf{0}}}\right)$ \\
\hline $\mathrm{x}=0$ & $\mathrm{Sr} 0$ & $5.21(1)$ & $2.71(1)$ \\
\hline $\mathrm{x}=0.1$ & $\mathrm{Sr} 1$ & $5.28(1)$ & $2.78(1)$ \\
\hline $\mathrm{x}=0.2$ & $\mathrm{Sr} 2$ & $5.38(1)$ & $2.88(1)$ \\
\hline $\mathrm{x}=0.3$ & $\mathrm{Sr} 3$ & $5.51(1)$ & $3.01(1)$ \\
\hline $\mathrm{x}=0.4$ & $\mathrm{Sr} 4$ & $5.54(1)$ & $3.04(1)$ \\
\hline $\mathrm{x}=0.5$ & $\mathrm{Sr} 5$ & $5.55(1)$ & $3.05(1)$ \\
\hline
\end{tabular}

The magnetic field dependence of magnetization $M(H)$ was characterized using a Lakeshore 7407 vibrating sample magnetometer using a maximum applied field of $15 \mathrm{kOe}$. For magnetocaloric studies, each sample was first heated in zero field to temperatures up to $350 \mathrm{~K}$, cooled in zero field to the measurement temperature and the subsequent $M(H)$ isotherms were measured with increasing field 
using a discontinuous protocol. Isothermal magnetization curves for samples $\mathrm{Sr} 0$ and $\mathrm{Sr} 1$ are shown in Figure 3.
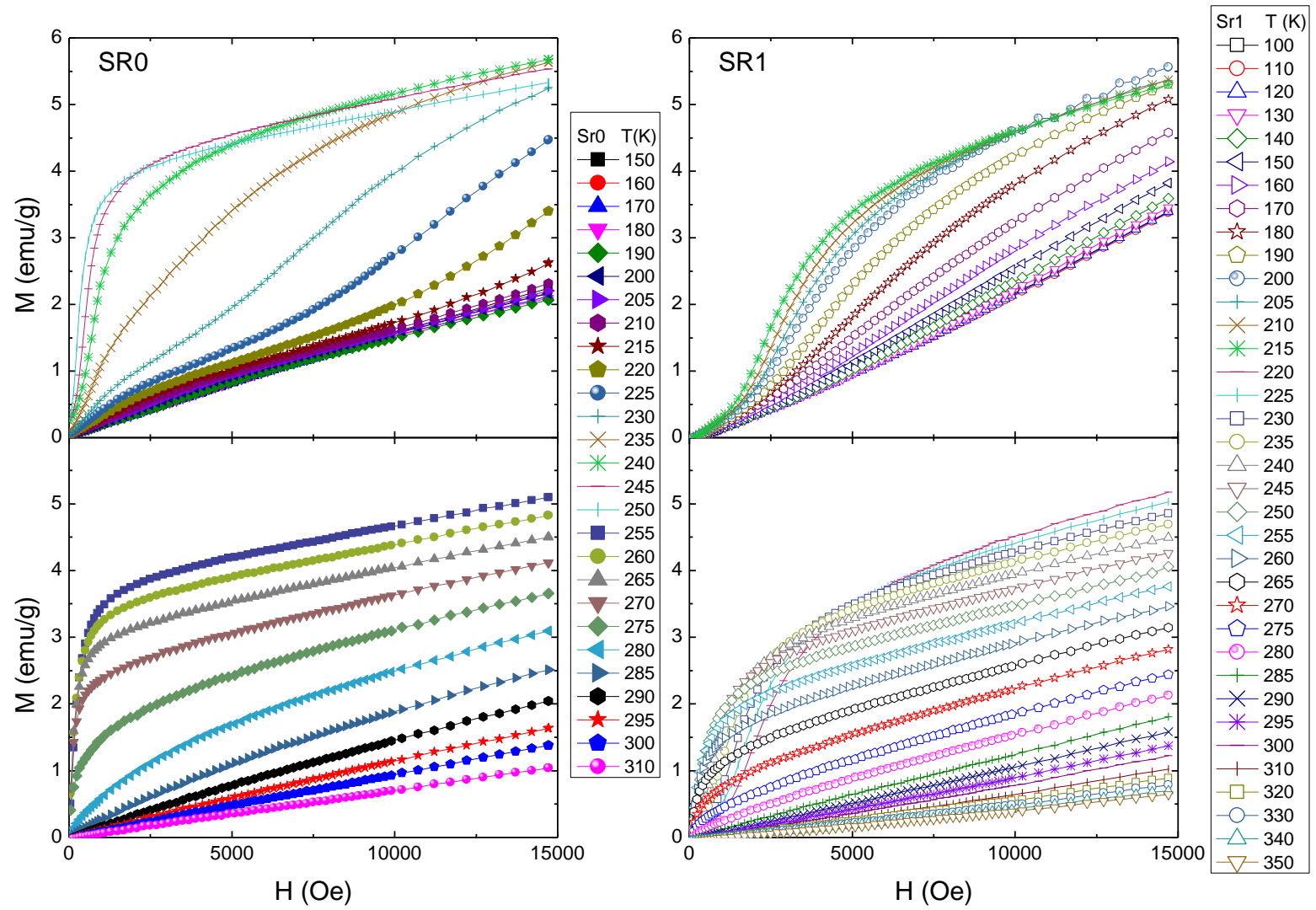

Fig. 3: Field dependence of magnetization measured at different temperatures for samples $\mathrm{Sr} 0$ and Sr1.

The magnetic entropy change $\left(\Delta S_{M}\right)$ due to the application of a magnetic field was calculated by:

$$
\Delta S_{M}=\mu_{0} \int_{0}^{H}\left(\frac{\partial M}{\partial T}\right)_{H} d H
$$

from the processing of the experimental temperature and field dependent magnetization data using the magnetocaloric effect analysis program [17] from LakeShore Cryotronics. The calculation of $\Delta S_{M}$ from this discontinuous measurement protocol avoids spurious results irrespective of the order of the phase transition $[17,18]$.

The magnetic field dependence of $\Delta S_{M}$ follows a power law expression [19]: 
$\Delta S_{M} \propto H^{n}$,

where its exponent $n$ (field and temperature dependent) can be locally calculated using:

$$
n=\frac{d \ln \left|\Delta S_{M}\right|}{d \ln H} .
$$

\section{Results and discussion}

The $M(T)$ zero field cooling and field cooling curves in Fig. 4 show that the $\mathrm{GdBa}_{1-\mathrm{x}} \mathrm{Sr}_{\mathrm{x}} \mathrm{Co}_{2} \mathrm{O}_{6-\delta}$ samples exhibit more than one magnetic phase transition. The pristine sample, SrO, (which crystallized in Pmmm orthorhombic symmetry with doubling in $b$ - and $c$ - axes) exhibits an AFM-FM transition around $240 \mathrm{~K}$ and a FM-PM transition around $275 \mathrm{~K}$. This leads to a narrow FM region observed for all the measured fields (up to $\sim 37 \mathrm{~K}$ for up to $500 \mathrm{Oe}$ ), which is analogous to results found in the literature [20, 21]. With minor $\mathrm{Sr}$ doping, $\mathrm{Sr} 1$ (maintaining the identical space group as undoped $\mathrm{Sr} 0$ at room temperature) shows a lowered transition temperature of AFM-FM $\left(T_{A F M-F M}\right)$ while retaining $T_{F M-P M}$. In this case, its FM window is expanded, as can be seen from the broader magnetization maximum in the main panel of Fig. 4. Furthermore, the abrupt slope at $T_{A F M-F M}$ observed in $\operatorname{Sr} 0$ converts into a gradual one (for $\operatorname{Sr} 1$ ) and was smeared out with higher Sr doping ( $\mathrm{x} \geq 0.2)$. Although $\mathrm{Sr} 2$ crystallizes in Pmmm symmetry (similar to $\mathrm{Sr} 0$ and $\mathrm{Sr} 1$ ), it retains only the doubling in $c$-axis (losing the doubling in $b$ ) and shows a lower $T_{F M-P M}$ as well as two smeared maxima at $\sim 240$ and $\sim 210 \mathrm{~K}$ when magnified (inset of Fig. 4). This could suggest another magnetic phase detected, which agrees with the structural analysis, wherein a 0.9 (2) wt.\% a single perovskite $\mathrm{GdCoO}_{3}$ was found by the Rietveld refinement of its XRD data and also observed by EPMA characterization[10]. For higher Sr content, a gradual decreasing magnetization with increasing temperature is observed, without AFM-FM this time, indicating a suppression of the latter with $\mathrm{Sr}^{2+}$ doping, similar to literature $[9,20,22]$. It is worth highlighting that the suppression was reported for higher oxygen content above $\mathrm{O}_{5.50}$ [9], wherein the authors considered it as a limiting composition corresponding to the type of doping carriers in $\mathrm{GdBaCo}_{2} \mathrm{O}_{5+\mathrm{y}}$ compounds: electron doping for $y<0.5$ and hole doping for $y>0.5$. Depending on the type of doping or, in other words, oxygen deficiency from $\mathrm{O}_{5.50}$, the $V_{C o}$ changes from $3+\left(\right.$ for $\left.\mathrm{O}_{5.50}\right)$ to a mixture of $2+/ 3+$ and $2+/ 3+/ 4+$ for $\mathrm{y}<0.5$ 
and $y>0.5$, respectively. This led to a noticeable alteration in the magnetic behavior as well as in conductivity in both regions. From Table 1, it is observed that the oxygen content exceeds this limiting composition (i.e. $>\mathrm{O}_{5.50}$ ) for $\mathrm{Sr}$ concentration of $\mathrm{x} \geq 0.3$, wherein the $A F M-F M$ phase transition was no longer observed in their $M(T)$ data unlike for lower Sr-containing samples (Fig. 4). In addition, an enhanced conductivity previously reported for our higher Sr-doped $\mathrm{GdBa}_{1-\mathrm{x}} \mathrm{Sr}_{\mathrm{x}} \mathrm{Co}_{2} \mathrm{O}_{6-\delta}$ samples $(\mathrm{Sr} 4)$ [10] exhibited an oxygen content above the $\mathrm{O}_{5.50}$ limiting composition, thus lays in the hole-doped region. This is in agreement with literature [9], wherein hole doping was attributed to the observed lowering of resistivity with increasing oxygen content above $\mathrm{O}_{5.50}$, (resistivity was lowered by $\sim 3$ orders of magnitude with $\mathrm{Sr}^{2+}$ mole fraction of $\mathrm{z}=0.07$ in $\mathrm{Gd}_{1-\mathrm{z}} \mathrm{Sr}_{\mathrm{z}} \mathrm{BaCo}_{2} \mathrm{O}_{5+\delta}[20]$ ). Furthermore, the authors suggested that an oxygen composition above $\mathrm{O}_{5.50}$ equates to the presence of $\mathrm{Co}^{4+}$ and proportional to hole doping. This is also observed for our higher Sr-containing samples, for e.g. Sr4 sample, which exhibits an average $V_{C o}$ of +3.04 , could exhibit some contributions of $\mathrm{Co}^{4+}$ to balance the $\mathrm{Co}^{2+}$ detected in XPS characterization [10].

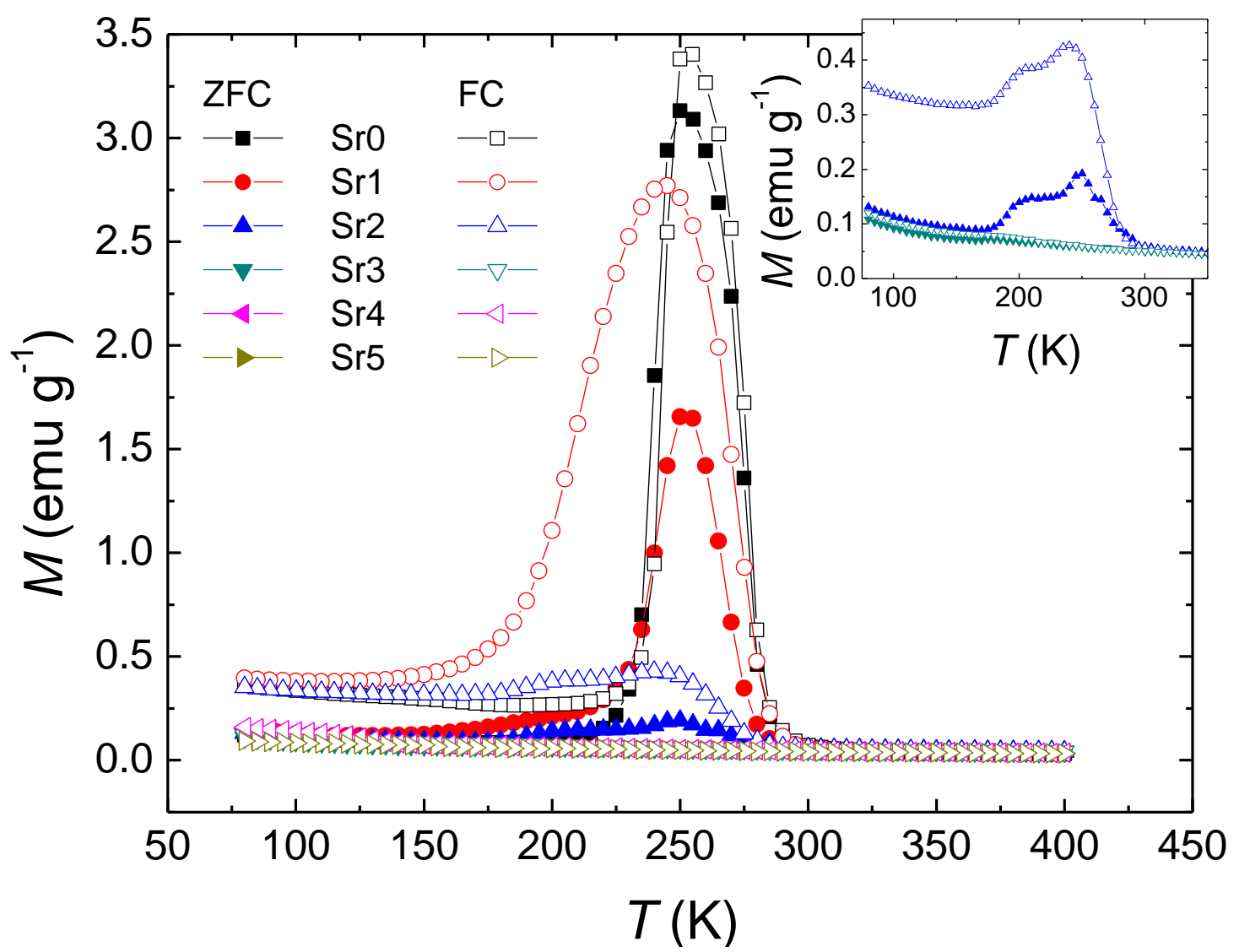


Fig. 4: Zero field cooling and field cooling curves of $\mathrm{GdBa}_{1-\mathrm{x}} \mathrm{Sr}_{\mathrm{x}} \mathrm{Co}_{2} \mathrm{O}_{6-\delta}$ : (main panel) whole sample series, and (inset) magnified views for $\mathrm{Sr} 2$ and $\mathrm{Sr} 3$. Applied field: $500 \mathrm{Oe}$.

Fig. 5 shows the field and temperature dependence of the magnetic entropy change for $\mathrm{Sr} 0$ and $\mathrm{Sr} 1$ samples. For higher amount of $\mathrm{Sr}^{2+}$ doping, the magnetocaloric response becomes negligible. Both of them feature inverse (positive $\Delta S_{M}$ ) MCE at low temperatures and closely accompanied by conventional MCE (negative $\Delta S_{M}$ ) at higher temperatures. To date, the $\mathrm{MCE}$ of $\mathrm{Gd}_{1-\mathrm{x}} \mathrm{Ca}_{\mathrm{x}} \mathrm{BaCo}_{2} \mathrm{O}_{5.5}$ is theoretically reported and models only considered its conventional MCE without the presence of its AFM-FM phase transition [15]. Their predictions of $\Delta S_{M}$ of an undoped sample $\left(\mathrm{GdBaCo}_{2} \mathrm{O}_{5.5}\right)$ coincide with the experimental values obtained in the present paper. For $\operatorname{Sr} 1$, the peak of the inverse $\operatorname{MCE}\left(\Delta S_{M \text { (inverse) }}^{\text {peak }}\right)$ and its corresponding temperature $\left(T_{p k}^{\text {inverse }}\right)$ reduce as compared to those of Sr0. Furthermore, the conventional MCE is observed to be more evident in Sr1 than in Sr0 sample, suggesting the stabilization of FM phase with $\mathrm{Sr}^{2+}$ doping and/or oxygen stoichiometry, consistent with their $M(T)$ results and also literature $[20,22]$.
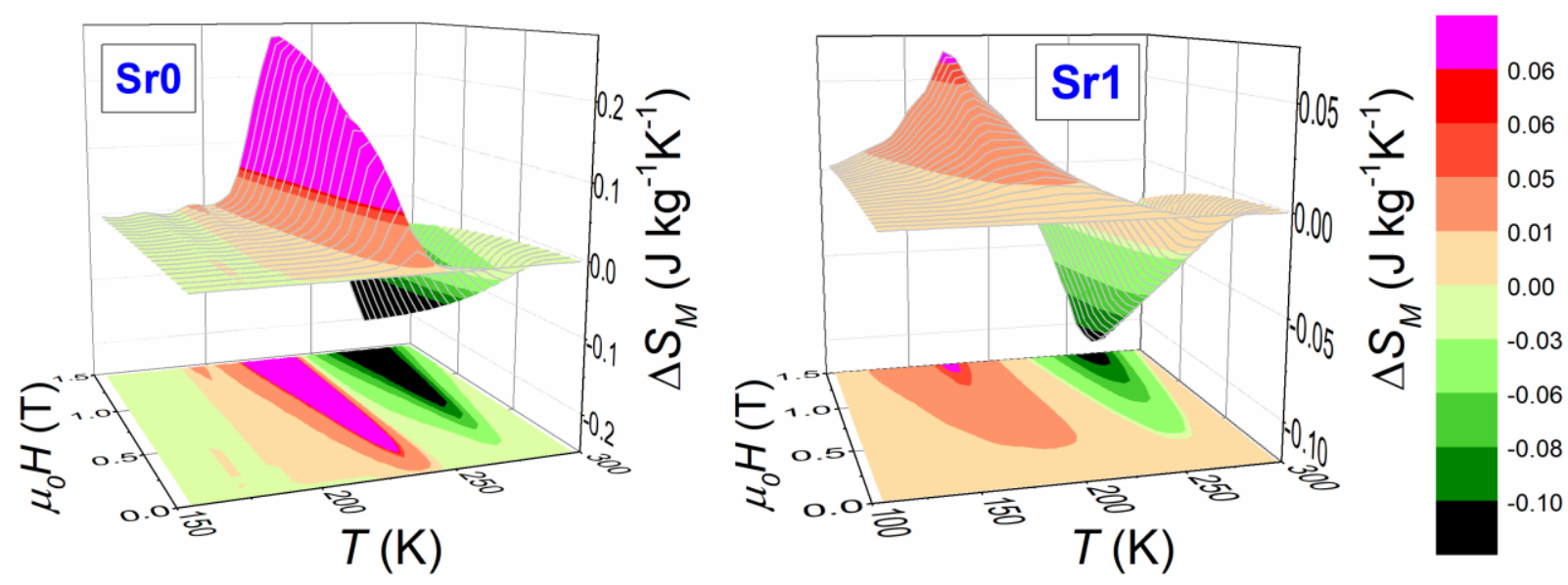

Fig. 5: Magnetic entropy change curves of $\mathrm{Sr} 0$ and $\mathrm{Sr} 1$ samples as a function of temperatures for all applied fields. The color map is also projected as bottom contour as a guide to the eye.

Fig. 6 shows a closer examination of $\Delta S_{M(\text { inverse })}^{\text {peak }}$ shifting to lower temperatures with magnetic fields ranging from 0.02 to $1.5 \mathrm{~T}$ as indicated with the arrows. Moreover, it can be observed that the flipping of the $\Delta S_{M}$ signs occurs earlier (i.e. at lower temperatures) with increasing magnetic fields, which 
indicates that the inverse MCE is compensated by the conventional MCE with increasing fields. Hence, on top of compositional effects, the competition of magnetic phases and the alteration of the order of the magnetic phase transition contributes to the magnitude of $\Delta S_{M}$ effected from its AFM-FM phase transition.
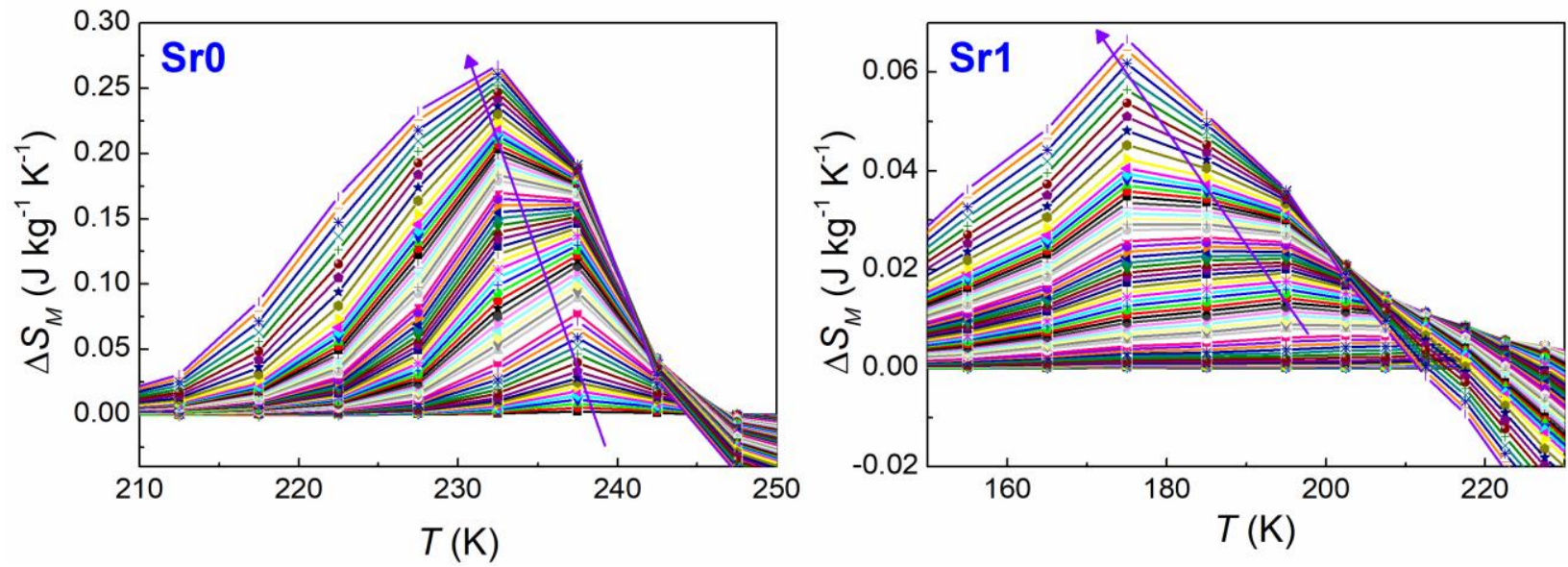

Fig. 6: Low temperature region of $\Delta S_{M \text { (inverse) }}(T)$ of $\mathrm{Sr} 0$ and $\mathrm{Sr} 1$ samples for 0.02-1.5 T. Arrows indicate the effect of increasing magnetic field.

Very recently, a quantitative criterion based on the magnetocaloric effect to determine the order of magnetic phase transition has been successfully applied to several magnetocaloric materials, namely La-Fe-Si-, Heusler-type alloys and composites, as well as numerical simulations[23]. As magnetocaloric materials are usually classified according to the order of their phase transitions (firstor second-order type), many have regarded that the ideal magnetocaloric material with a large effect with minimal or without hysteresis would be in-between the two classifications (at the region termed as the critical point of second-order phase transition) [11]. Hence, it is interesting to apply this recently proposed quantitative criterion to our work for evaluation, whereby the technique could distinguish the classifications even for compositions near the critical point of second-order phase transition without being confined to a particular model but based on the magnetic field dependence of $\Delta S_{M}$ (equations 2 and 3) [24]. Fig. 7(a) shows the 2D plots of $\Delta S_{M}(T)$ for $\mathrm{Sr} 0$ with applied fields up to $1.5 \mathrm{~T}$. The dashed green line corresponds to $\Delta S_{M}=0$ and inverse and conventional MCE are indicated by the blue and pink regions, respectively. The darker shades of the blue and pink regions illustrate increasing magnetic 
fields. The $T_{p k}^{\text {inverse }}$ shifts to lower temperatures with increasing fields while $T_{p k}^{\text {conventional }}$ remains at approximately the same position as a function of field. For Fig. 7(b), the exponent $n$ from the field dependence of $\Delta S_{M}$ for the $\operatorname{Sr} 0$ sample is presented as a function of temperature. At low temperatures, an overshoot of $n>2$ (illustrated by the blue regions) is observed near the onset of $T_{A F M-F M}$ (within the region of 212-222 K as they are field dependent), indicating a first-order phase transition (FOPT), which is in agreement with the order of the phase transition indicated in literature [25-27]. These overshoots shift to lower temperatures with increasing field, in agreement with the field dependent $T_{p k}^{\text {inverse }}$ observed earlier. The characteristic spikes observed near $242-248 \mathrm{~K}$ (yellow region) indicate the switching of signs of $\Delta S_{M}$ from inverse to conventional MCE (indicated by the green dotted line which correspond to $\Delta S_{M}=0$ ), analogous with the $n(T)$ behavior of a Heusler alloy reported in ref.[23]. The exponent $n$ then goes to a minimum (corresponding to the Curie temperature of the FM-PM phase transition) prior to approaching a value of up to 2 (paramagnetic region), which agrees with the $n(T)$ behavior of a SOPT (shown in shades of pink in Fig. 7) [19]. 


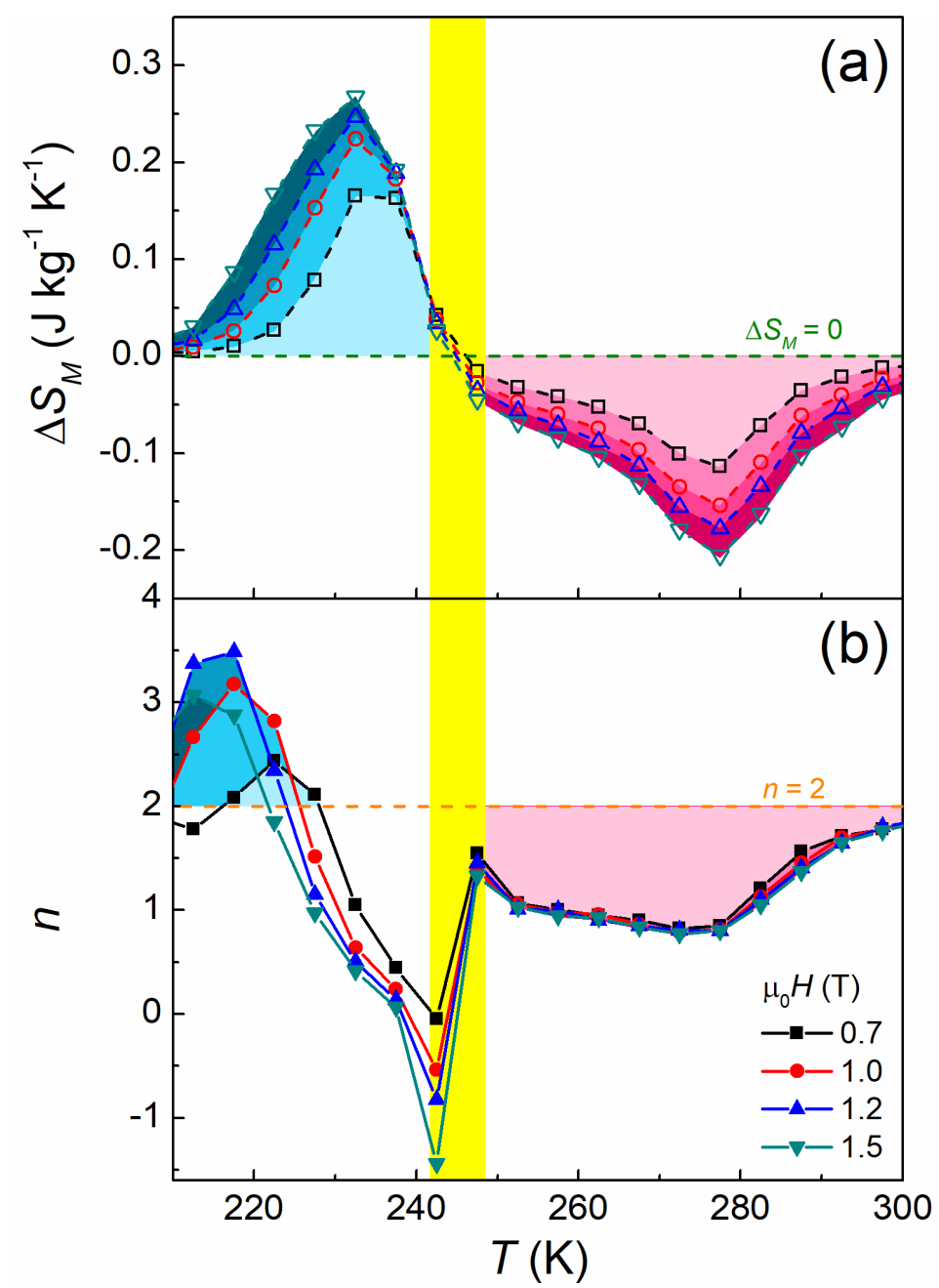

Fig. 7: Temperature and field dependence of: (a) $\Delta S_{M}$ and (b) exponent $n$ for Sr0 sample at different applied fields. Dash green line corresponds to $\Delta S_{M}=0$ in (a) while the orange indicates $n=2$ in (b). Inverse and conventional MCE are indicated by the blue and pink regions in (a), respectively. The yellow region corresponds to a change in the sign of $\Delta S_{M}$, producing a spike in $n$.

The field dependence of $\Delta S_{M}$ and exponent $n$ as a function of temperature for $\operatorname{Sr} 1$ at $\mu_{0} H=1.5 \mathrm{~T}$ are presented in Fig. 8. Fig. 8(a) shows that the sample exhibits an inverse MCE (blue region) at low temperatures and a conventional MCE (pink region) at higher temperatures. On the other hand, its $n(T)$ plot in Fig. 8(b) shows only the characteristic spikes of switching MCE signs but without any overshoot of $n>2$. Its exponent $n$ was observed to first begin at $\sim 1.6$ (no blue shaded region this time unlike for Sr0 in Fig. 7(b)) at low temperatures then display characteristic spikes (yellow region) of switching MCE signs at approximately $207-213 \mathrm{~K}$. With increasing temperatures thereafter, $n$ tends to a minimum at the Curie temperature of FM-PM before approaching a value of up to $2(T>300 \mathrm{~K})$ as 
illustrated in the pink region in Fig. 8(b). These observations reveal that though the minor doping of $\mathrm{Sr}^{2+}$ in $\mathrm{GdBa}_{1-\mathrm{x}} \mathrm{Sr}_{\mathrm{x}} \mathrm{Co}_{2} \mathrm{O}_{6-\delta}$ does not suppress the AFM-FM phase transition at low temperatures, it considerably transformed FOPT-character into a SOPT-type, which is not obvious from the $\Delta S_{M}(T)$ data.

The change in the order of the phase transition from FOPT to SOPT decreases considerably the magnetic entropy change of the perovskite. However, this change is produced without altering the crystalline structure at room temperature or the type of doping carrier for cobaltites (both $\mathrm{Sr} 0$ and $\mathrm{Sr} 1$ samples lay in the electron-doped category based on their oxygen content).

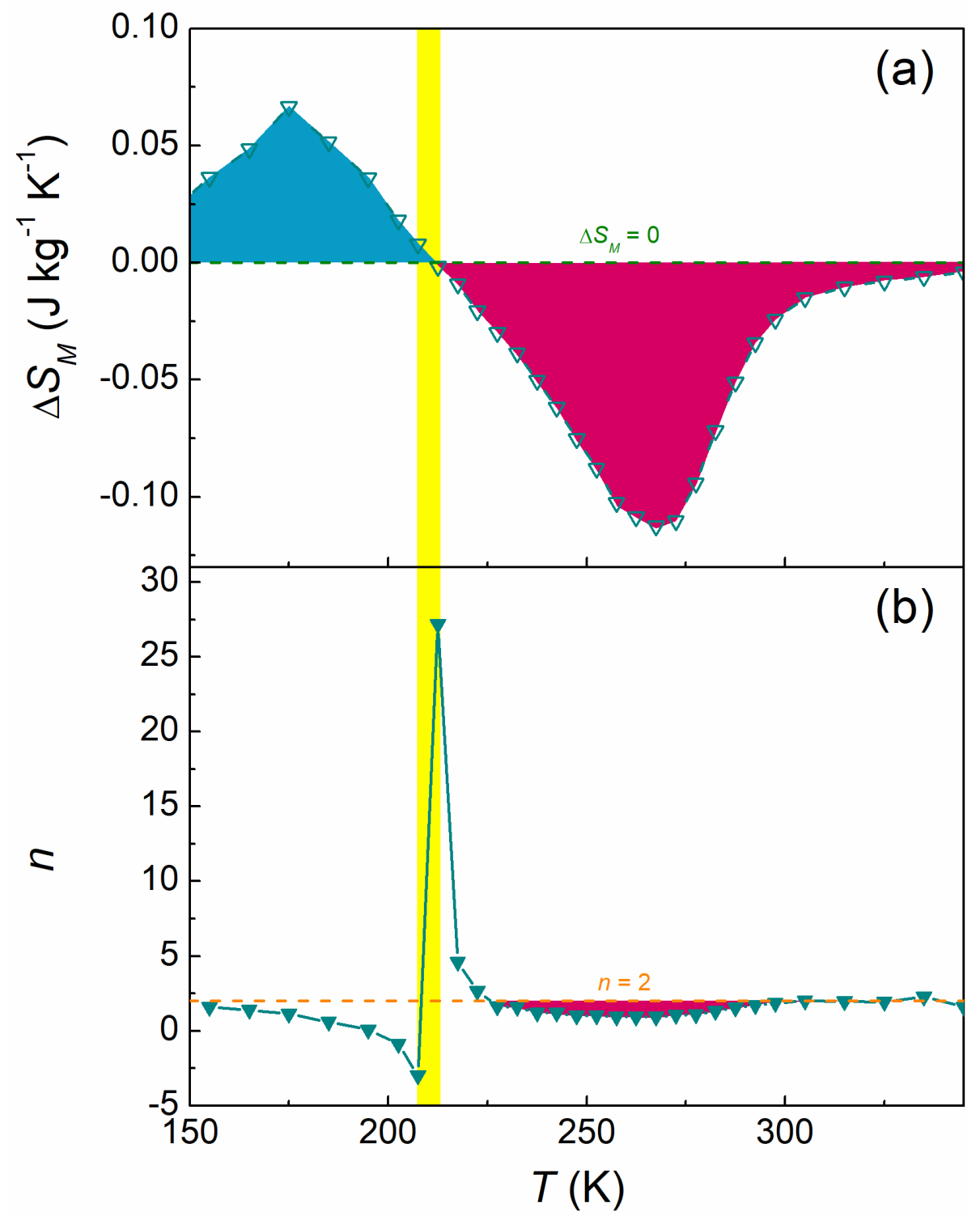


Fig. 8: Temperature dependence of: (a) $\Delta S_{M}$ and (b) exponent $n$ for $\mathrm{Sr} 1$ sample at a single field of 1.5 $\mathrm{T}$. Inverse and conventional MCE are indicated by the blue and pink regions in (a), respectively. The yellow region corresponds to a change in the sign of $\Delta S_{M}$, producing a spike in $n$.

Furthermore, the universal curve method is another way to distinguish FOPT or SOPT based on the presence or absence of a qualitative feature, and has been widely used and applicable to identify the type of magnetocaloric materials[19, 28]. Its construction procedure details can be found in ref.[19]. In short, the magnetic entropy change curves of the samples were normalized and their temperature axes were phenomenologically rescaled, which are presented in Fig. 9 for $\mathrm{Sr} 0$ and $\mathrm{Sr} 1$. For $\mathrm{Sr} 0$ at low temperatures (i.e. AFM-FM) in Fig. 9(a), data do not collapse onto a universal curve, indicating a FOPT in the AFM-FM transition, which is in agreement with Fig. 7. However, a good collapse onto a single universal curve observed at higher temperatures in Fig. 9(b) indicates that Sr0 undergoes a SOPT in the FM-PM region. On the other hand, Sr1 shows a good collapse of data at both AFM-FM and FM-PM temperature regions (Figs. 9(c) and (d)), suggesting that both have undergone SOPT. These observations reinforce with the analyses from Figs. 7 and 8, affirming that the FOPT-character of the AFM-FM of Sr1 sample was lost, and they prove the applicability of the quantitative criterion to cobaltites. 

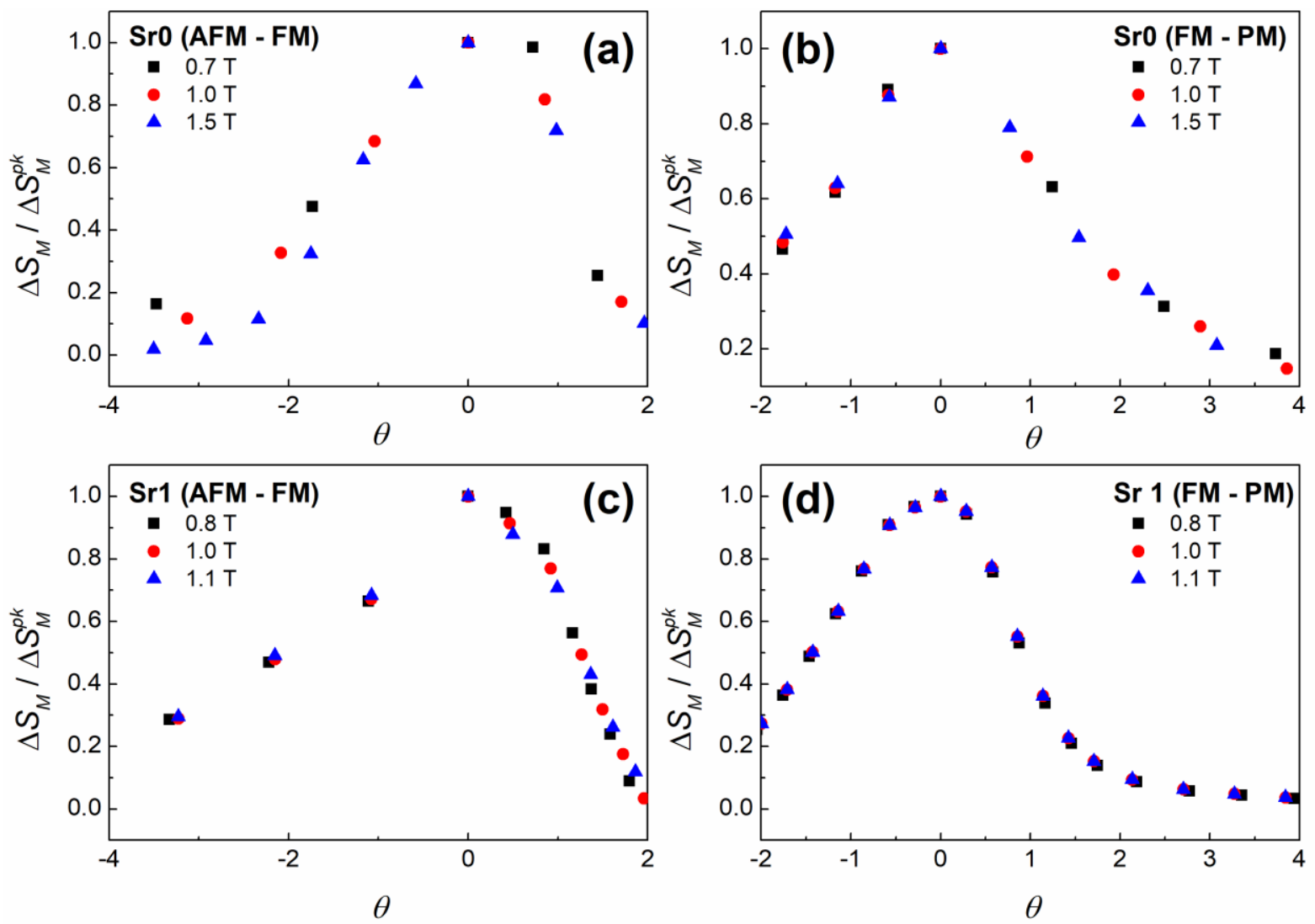

Fig. 9: Rescaling of the magnetic entropy change curves of $\mathrm{Sr} 0$ and $\mathrm{Sr} 1$ for different applied magnetic fields: (a, b) and (c, d) respectively.

It is worth mentioning that the presence of very minor hysteresis in the temperature dependent magnetic entropy change curves can be misleading when using it to identify the order of the phase transition[29], inasmuch as thermal lag of the experimental setup might lead to erroneous conclusions. Therefore, that criterion is avoided in the current paper.

\section{Conclusions}

The properties of cobaltites are known to be highly dependent on their oxygen content deviating from the critical limit of $\mathrm{O}_{5.50}$ leading to different type of doping carriers: electron-doped for $\delta<0.5$ while holedoped for $\delta>0.5$. The electron-doped $\mathrm{GdBa}_{1-\mathrm{x}} \mathrm{Sr}_{x} \mathrm{Co}_{2} \mathrm{O}_{6-\delta}$ double perovskite samples correspond to $\mathrm{Sr}$ additions of up to $\mathrm{x}=0.2$ while higher $\mathrm{Sr}$ concentration led to hole doping by oxygen additions $(\delta>0.5)$. Their thermomagnetic behavior were observed to fall into two systems: for electron-doped samples, the pristine ( $\mathrm{Sr} 0), \mathrm{Sr} 1$ and $\mathrm{Sr} 2$ samples present a AFM-FM phase transition at low temperatures and a FMPM transition at higher temperatures, though the former phase transition is very much smeared out for $\mathrm{Sr} 2$ sample. Conversely, only a gradual FM-PM transition is observed for hole-doped samples $(\mathrm{Sr} 3$ - 
Sr5) while the AFM-FM is suppressed. In addition, $\mathrm{Sr} 2$ and $\mathrm{Sr} 3$ samples lost the doubling in the $b$-axis though retaining the doubling in $c$ - and crystallographic symmetry $(P m m m)$ while $\mathrm{Sr} 4$ and $\mathrm{Sr} 5$ completely adopt a different space group $(P 4 / \mathrm{mmm})$.

The pristine sample was shown to present an inverse and a conventional magnetocaloric effect arising from its AFM-FM transition and FM-PM transition respectively. The exponent $n$ of its magnetic field dependence of $\Delta S_{M}$ shows an overshoot of $n>2$, which shifts to lower temperatures with field, consistent with the field dependence of transition temperature of its AFM-FM (which decreases with field). By using the recently proposed quantitative criterion for identifying the order of phase transitions based on the magnetocaloric effect, the fingerprint of the overshoot of $n>2$ clearly indicates that its AFM-FM is a first-order phase transition. On the other hand for Sr1 sample, though with minor doping of $x=0.1$ and retaining the same room temperature crystallographic symmetry as that of Sr0 sample (Pmmm with doubling in $b$ and $c$ axes), its $n$ behavior uncovers that the first-order nature of AFM-FM was transformed into a second-order-type despite that it still exhibits a similar-looking thermomagnetic behavior and inverse magnetocaloric effect as that of the pristine sample. This was only clearly demonstrated upon further evaluation of its magnetocaloric behavior using the universal curve and magnetic field dependence methods. Therefore, it is evident that doped samples that retain the same crystal structure and electron-doped carrier as the undoped composition might exhibit remarkably different magnetic properties due to the change of the order of phase transition.

\section{Acknowledgments}

This work was supported by AEI/FEDER-UE (project MAT-2016-77265-R) and the PAI of the Regional Government of Andalucía. S. S. P. and S. J. S. also acknowledge the support of the EPSRC (EP/M014142/1).

\section{References}

[1] S. Yáñez-Vilar, A. Castro-Couceiro, B. Rivas-Murias, A. Fondado, J. Mira, J. Rivas, M.A. Señarís-Rodríguez, Study of the Dielectric Properties of the Perovskite LaMn0.5Co0.5O3- $\delta, Z$ Anorg Allg Chem, 631 (2005) 2265-2272.

[2] Z. Shao, S.M. Haile, A high-performance cathode for the next generation of solid-oxide fuel cells, Nature, 431 (2004) 170. 
[3] M. Yashima, Diffusion pathway of mobile ions and crystal structure of ionic and mixed conductors $\langle$ br $>$ - A brief review, Journal of the Ceramic Society of Japan, 117 (2009) 1055-1059.

[4] S. Zhou, X. Miao, X. Zhao, C. Ma, Y. Qiu, Z. Hu, J. Zhao, L. Shi, J. Zeng, Engineering electrocatalytic activity in nanosized perovskite cobaltite through surface spin-state transition, Nature Communications, 7 (2016) 11510.

[5] Y. Tong, Y. Guo, P. Chen, H. Liu, M. Zhang, L. Zhang, W. Yan, W. Chu, C. Wu, Y. Xie, SpinState Regulation of Perovskite Cobaltite to Realize Enhanced Oxygen Evolution Activity, Chem, 3 (2017) 812-821.

[6] R. Mahendiran, A.K. Raychaudhuri, Magnetoresistance of the spin-state-transition compound La-xSrxCoO3, Phys Rev B, 54 (1996) 16044-16052.

[7] N. Khan, A. Midya, K. Mydeen, P. Mandal, A. Loidl, D. Prabhakaran, Critical behavior in singlecrystallineLa0.67Sr0.33CoO3, Physical Review B, 82 (2010) 064422.

[8] V.B. Shenoy, C.N.R. Rao, Electronic phase separation and other novel phenomena and properties exhibited by mixed-valent rare-earth manganites and related materials, Philosophical Transactions of the Royal Society A: Mathematical, Physical and Engineering Sciences, 366 (2008) 63.

[9] A.A. Taskin, A.N. Lavrov, Y. Ando, Transport and magnetic properties ofGdBaCo2O5+xsingle crystals: A cobalt oxide with square-latticeCoO2planes over a wide range of electron and hole doping, Physical Review B, 71 (2005) 134414.

[10] S.S. Pramana, A. Cavallaro, C. Li, A.D. Handoko, K.W. Chan, R.J. Walker, A. Regoutz, J.S. Herrin, B.S. Yeo, D.J. Payne, J.A. Kilner, M.P. Ryan, S.J. Skinner, Crystal structure and surface characteristics of Sr-doped GdBaCo2O6- $\delta$ double perovskites: oxygen evolution reaction and conductivity, Journal of Materials Chemistry A, 6 (2018) 5335-5345.

[11] V. Franco, J.S. Blázquez, J.J. Ipus, J.Y. Law, L.M. Moreno-Ramírez, A. Conde, Magnetocaloric effect: From materials research to refrigeration devices, Prog Mater Sci, 93 (2018) 112-232.

[12] F. Saadaoui, R. M'Nassri, A. Mleiki, M. Koubaa, N.C. Boudjada, A. Cheikhrouhou, Studies on the structure, critical behavior and magnetocaloric effect in ( $\mathrm{LaBi}) \mathrm{SrCoO}$ cobaltite, J. Mater. Sci.Mater. Electron., 28 (2017) 15500-15511.

[13] I.G. Deac, A. Vladescu, Magnetic and magnetocaloric properties of $\mathrm{Pr} 1-\mathrm{xSrxCoO} 3$ cobaltites, J Magn Magn Mater, 365 (2014) 1-7.

[14] M.S. Reis, D.L. Rocco, R.J. Caraballo Vivas, B. Pimentel, N.R. Checca, R. Torrão, L. Paixão, A.M. dos Santos, Spin state and magnetic ordering of half-doped Nd0.5Sr0.5CoO3 cobaltite, J Magn Magn Mater, 422 (2017) 197-203.

[15] M.A. Hamad, Magnetocaloric effect in polycrystalline $\mathrm{Gd}_{1-\mathrm{x}} \mathrm{Ca}_{x} \mathrm{BaCo}_{2} \mathrm{O}_{5.5}$, Mater Lett, 82 (2012) 181-183.

[16] P. Kumar, R. Mahendiran, Magnetothermopower, magnetic entropy change, and magnetoresistance in ferromagnetic cobaltites: R0.7Sr0.3CoO3 ( $\mathrm{R}=\mathrm{La}$ and $\mathrm{Gd})$, Appl Phys Lett, 106 (2015) 142401.

[17] V. Franco, Determination of the Magnetic Entropy Change from Magnetic Measurements: the Importance of the Measurement Protocol, in, Lake Shore Cryotronics, http://www.lakeshore.com/products/Vibrating-Sample-Magnetometer/Pages/MCE.aspx, 2014. 
[18] B. Kaeswurm, V. Franco, K.P. Skokov, O. Gutfleisch, Assessment of the magnetocaloric effect in $\mathrm{La}, \mathrm{Pr}(\mathrm{Fe}, \mathrm{Si})$ under cycling, J Magn Magn Mater, 406 (2016) 259-265.

[19] V. Franco, A. Conde, Scaling laws for the magnetocaloric effect in second order phase transitions: From physics to applications for the characterization of materials, Int J Refrig, 33 (2010) 465-473.

[20] J. Janaki, A. Bharathi, N. Gayathri, P. Yasodha, M. Premila, V.S. Sastry, Y. Hariharan, Sr2+ doping effects on the transport and magnetic properties of $\mathrm{GdBaCo} 2 \mathrm{O} 5+\delta$, Physica $\mathrm{B}, 403$ (2008) 631-635.

[21] I.O. Troyanchuk, N.V. Kasper, D.D. Khalyavin, H. Szymczak, R. Szymczak, M. Baran, Magnetic and electrical transport properties of orthocobaltites R0.5Ba0.5CoO3, Phys Rev B, 58 (1998) 2418-2421.

[22] N. Thirumurugan, A.T. Satya, J. Janaki, A. Bharathi, Effect Of Hole Doping On Magnetic Properties Of GdBaCo2O5.5, AIP Conference Proceedings, 1347 (2011) 274-277.

[23] J.Y. Law, V. Franco, L.M. Moreno-Ramírez, A. Conde, D.Y. Karpenkov, I. Radulov, K.P. Skokov, O. Gutfleisch, A quantitative criterion for determining the order of magnetic phase transitions using the magnetocaloric effect, Nature Communications, 9 (2018) 2680.

[24] L.M. Moreno-Ramírez, C. Romero-Muñiz, J.Y. Law, V. Franco, A. Conde, I.A. Radulov, F. Maccari, K.P. Skokov, O. Gutfleisch, The role of Ni in modifying the order of the phase transition of $\mathrm{La}(\mathrm{Fe}, \mathrm{Ni}, \mathrm{Si}) 13$, Acta Mater, 160 (2018) 137-146.

[25] I.O. Troyanchuk, N.V. Kasper, D.D. Khalyavin, H. Szymczak, R. Szymczak, M. Baran, Phase Transitions in the Gd0.5Ba0.5CoO3 Perovskite, Phys Rev Lett, 80 (1998) 3380-3383.

[26] D.D. Khalyavin, S.N. Barilo, S.V. Shiryaev, G.L. Bychkov, I.O. Troyanchuk, A. Furrer, P. Allenspach, H. Szymczak, R. Szymczak, Anisotropic magnetic, magnetoresistance, and electrotransport properties of GdBaCo2O5.5 single crystals, Phys Rev B, 67 (2003) 214421.

[27] C. Martin, A. Maignan, D. Pelloquin, N. Nguyen, B. Raveau, Magnetoresistance in the oxygen deficient LnBaCo2O5.4 (Ln=Eu, Gd) phases, Appl Phys Lett, 71 (1997) 1421-1423.

[28] C. Romero-Muñiz, V. Franco, A. Conde, Two different critical regimes enclosed in the BeanRodbell model and their implications for the field dependence and universal scaling of the magnetocaloric effect, Phys Chem Chem Phys, 19 (2017) 3582-3595.

[29] V. Franco, J.Y. Law, A. Conde, V. Brabander, D.Y. Karpenkov, I. Radulov, K. Skokov, O. Gutfleisch, Predicting the tricritical point composition of a series of LaFeSi magnetocaloric alloys via universal scaling, Journal of Physics D: Applied Physics, 50 (2017) 414004. 


\section{Table Captions}

Table 1. Oxygen content and cobalt valencies in $\mathrm{GdBa}_{1-\mathrm{x}} \mathrm{Sr}_{\mathrm{x}} \mathrm{Co}_{2} \mathrm{O}_{6-\delta}$

\begin{tabular}{|c|c|c|c|}
\hline Sr content $(\mathbf{x})$ & Sample & Oxygen content $(6-\delta)$ & Cobalt Valency $\left(\mathbf{V}_{\mathbf{C}_{\mathbf{0}}}\right)$ \\
\hline $\mathrm{x}=0$ & $\mathrm{Sr} 0$ & $5.21(1)$ & $2.71(1)$ \\
\hline $\mathrm{x}=0.1$ & $\mathrm{Sr} 1$ & $5.28(1)$ & $2.78(1)$ \\
\hline $\mathrm{x}=0.2$ & $\mathrm{Sr} 2$ & $5.38(1)$ & $2.88(1)$ \\
\hline $\mathrm{x}=0.3$ & $\mathrm{Sr} 3$ & $5.51(1)$ & $3.01(1)$ \\
\hline $\mathrm{x}=0.4$ & $\mathrm{Sr} 4$ & $5.54(1)$ & $3.04(1)$ \\
\hline $\mathrm{x}=0.5$ & $\mathrm{Sr} 5$ & $5.55(1)$ & $3.05(1)$ \\
\hline
\end{tabular}

\section{Figure Captions}

Fig. 1: Crystal structure representation of (a) a single perovskite $\mathrm{ABO}_{3}$, where $\mathrm{A}$ (green) is located in the centre of corner-connected $\mathrm{BO}_{6}$ octahedra (blue); (b) an ordered perovskite $\mathrm{AA}^{\prime} \mathrm{B}_{2} \mathrm{O}_{6}$, where $\mathrm{A}$ (green) and $\mathrm{A}^{\prime}$ (purple) stack in an alternate sequence along z-crystallographic axis with cornerconnected BO6 octahedra shown in brown, for example $\mathrm{GdBaCo}_{2} \mathrm{O}_{6}$; (c) defective ordered perovskite $\mathrm{AA}^{\prime} \mathrm{B}_{2} \mathrm{O}_{6-\delta}$, where the vacancy of oxygen $(\delta$, red) is preferentially created in the $x y$-plane in the A' layer.

Fig. 2: Rietveld refinement of the XRD data of $\mathrm{Sr} 1$ sample performed at room temperature using $\mathrm{Cu} K_{\alpha}$ radiation $\left(R_{w p}=0.029, R_{\text {Bragg }}=0.009\right)$. The doubling in its $c$-axis is indicated by $2 \theta_{001}=11.73^{\circ}$ and its doubling in $b$ by $2 \theta_{010}=11.33^{\circ}, 2 \theta_{130}=41.79^{\circ}$ and $2 \theta_{131}=43.56^{\circ}$.

Fig. 3: Field dependence of magnetization measured at different temperatures for samples $\mathrm{Sr} 0$ and Sr1.

Fig. 4: Field cooling curves of $\mathrm{GdBa}_{1-\mathrm{x}} \mathrm{Sr}_{\mathrm{x}} \mathrm{Co}_{2} \mathrm{O}_{6-8}$ : (main panel) whole sample series, and (inset) magnified views for Sr2 and Sr3. Applied field: 500 Oe.

Fig. 5: Magnetic entropy change curves of $\mathrm{Sr} 0$ and $\mathrm{Sr} 1$ samples as a function of temperatures for all applied fields. The colormap is also projected as bottom contour as a guide to the eye.

Fig. 6: Low temperature region of $\Delta S_{M \text { (inverse) }}(T)$ of Sr0 and Sr1 samples for 0.02-1.5 T.

Fig. 7: Temperature and field dependence of: (a) $\Delta S_{M}$ and (b) exponent $n$ for Sr0 sample at different applied fields. Dash line correspond to $\Delta S_{M}=0$. Inverse and conventional MCE are indicated by the blue and pink regions in (a), respectively. The yellow region corresponds to a change in the sign of $\Delta S_{M}$, producing a spike in $n$.

Fig. 8: Temperature dependence of: (a) $\Delta S_{M}$ and (b) exponent $n$ for Sr1 sample at single field of $1.5 \mathrm{~T}$. Inverse and conventional MCE are indicated by the blue and pink regions in (a), respectively. The yellow region corresponds to a change in the sign of $\Delta S_{M}$, producing a spike in $n$.

Fig. 9: Rescaling of the magnetic entropy change curves of $\mathrm{Sr} 0$ and $\mathrm{Sr} 1$ for different applied magnetic fields: (a, b) and (c, d) respectively. 